\title{
MICROLOCAL ANALYSIS OF THE BOCHNER-MARTINELLI INTEGRAL
}

\author{
NIKOLAI TARKHANOV AND NIKOLAI VASILEVSKI
}

(Communicated by XXX)

\begin{abstract}
In order to characterise the $C^{*}$-algebra generated by the singular Bochner-Martinelli integral over a smooth closed hypersurfaces in $\mathbb{C}^{n}$, we compute its principal symbol. We show then that the Szegö projection belongs to the strong closure of the algebra generated by the singular Bochner-Martinelli integral.
\end{abstract}

\section{CONTEnTs}

1. Introduction

2. Singular Bochner-Martinelli integral 2

3. Evaluation of the symbol 4

4. $C^{*}$-algebra 6

5. The Szegö projection $\quad 7$

References $\quad 9$

\section{INTRODUCTION}

The Bochner-Martinelli integral formula for holomorphic functions in a bounded domain in $\mathbb{C}^{n}$ is of great importance in complex analysis, cf. [5]. It is a generalization to many variables of the classical Cauchy formula which actually gave rise to the theory of singular integral equations. A canonical Cauchy-type singular integral known as Hilbert transform is a corner stone of harmonic analysis. To handle more refined integral operators of many-dimensional complex analysis, such as CauchyFantappiè integrals or Szegö projections, etc., there have been elaborated several calculi of pseudodifferential operators relevant to several complex variables, cf. [7].

The Bochner-Martinelli integral does not apply to derive explicit formulas for a solution of the $\bar{\partial}$-equation, which is a fundamental equation of complex analysis. On the other hand, the singular Bochner-Martinelli integral over each smooth hypersurface satisfies the cancellation condition, and thus defines a singular integral operator on the hypersurface. In other words, it belongs to the algebra of

Received by the editors November 12, 2005.

2000 Mathematics Subject Classification. Primary 32A25; Secondary 47L15, 47G30.

Key words and phrases. The Bochner-Martinelli integral, symbol, $C^{*}$-algebra, Szegö projection. 
pseudodifferential operators of order zero with polyhomogeneous symbols. Since its kernel is very explicit the problems of complex analysis have never required, as far as we know, the knowledge of its symbol but in the case $n=1$. The analysis of the Bochner-Martinelli singular integral so far undertaken does not go beyond the potential theory of the 1950s, cf. [4].

Since explicit kernels are more useful than the Fourier transform under the presence of singularities of the underlying hypersurface, the existing theory of the Bochner-Martinelli integral leads to the conclusion that no pseudodifferential technique is required in this theory at all. However, the theory of operator algebras gives us an evidence to the contrary. To effectively describe the $C^{*}$-algebra generated by the singular Bochner-Martinelli integral the knowledge of its explicit kernel is obviously insufficient, for the composition rule for the kernels includes integration over the hypersurface and cannot be carried out explicitly. On the other hand, the composition rule for the principal symbols allows one to evaluate the principal symbol of a power of the singular Bochner-Martinelli integral explicitly. This immediately gives rise to the Calkin algebra of the algebra under study.

The aim of this paper is to bring together two areas whose interaction might enrich each other. The first of the two is the theory of algebras of pseudodifferential operators with symbol structure. And the second area is the potential theory of the Bochner-Martinelli kernel, which applies to many central problems of complex analysis.

\section{Singular Bochner-Martinelli integral}

Let $S$ be a smooth closed hypersurface in $\mathbb{C}^{n}$, where $n \geq 1$. The surface measure $d s$ on $S$ is induced by the Lebesgue measure $d y=d y_{1} \wedge \ldots \wedge d y_{2 n}$ in $\mathbb{R}^{2 n}$, where the complex structure is introduced by $\zeta_{j}=y_{j}+\imath y_{n+j}$, for $j=1, \ldots, n$. A trivial verification shows that $d y=(2 \imath)^{-n} d \bar{\zeta} \wedge d \zeta$, where $d \zeta=d \zeta_{1} \wedge \ldots \wedge d \zeta_{n}$ and similarly for $d \bar{\zeta}$.

If $S$ is given in the form $S=\left\{\zeta \in \mathbb{C}^{n}: \varrho(\zeta)=0\right\}$, where $\varrho \in C^{1}\left(\mathbb{C}^{n}\right)$ is a real-valued function satisfying $\nabla \varrho(\zeta) \neq 0$ for all $\zeta \in S, \nabla \varrho(\zeta)$ standing for the real gradient of $\varrho$ at $\zeta$, then

$$
\nu(\zeta)=\frac{\nabla \varrho(\zeta)}{|\nabla \varrho(\zeta)|}
$$

is the unit normal vector of $S$ at a point $\zeta \in S$ oriented in the direction of increasing of $\rho$. The complex vector $\nu_{c}=\left(\nu_{c, 1}, \ldots, \nu_{c, n}\right)$ with coordinates $\nu_{c, j}=\nu_{j}+\imath \nu_{n+j}$ is called the complex normal of the hypersurface $S$. In the coordinates of $\mathbb{C}^{n}$ we obviously have

for $j=1, \ldots, n$.

$$
\nu_{c, j}(\zeta)=\frac{\frac{\partial \varrho}{\partial \bar{\zeta}_{j}}}{\left|\nabla_{\bar{\zeta}} \varrho(\zeta)\right|}
$$
$d \bar{\zeta}_{j}$.

We now denote by $d \bar{\zeta}[j]$ the wedge product of all differentials $d \bar{\zeta}_{1}, \ldots, d \bar{\zeta}_{n}$ but

Lemma 2.1. For each $j=1, \ldots, n$, the pull-back of the differential form $d \zeta \wedge d \bar{\zeta}[j]$ under the embedding $S \hookrightarrow \mathbb{C}^{n}$ is equal to $(-1)^{j-1}(2 \imath)^{n-1} \imath \nu_{c, j} d s$, where ds is the surface measure on $S$. 
Proof. An easy computation shows that the pull-back of the differential form $d y[j]$ under the embedding $S \hookrightarrow \mathbb{R}^{2 n}$ is equal to $(-1)^{j-1} \nu_{j} d s$, for every $j=1, \ldots, 2 n$. From this the lemma follows immediately.

Given an integrable function $f: S \rightarrow \mathbb{C}$ with compact support on $S$, the BochnerMartinelli integral of $f$ is defined by

$$
M f(z)=\int_{S} f(\zeta) U(\zeta, z)
$$

for $z \notin S$, where

$$
U(\zeta, z)=\frac{(n-1) !}{(2 \pi \imath)^{n}} \sum_{j=1}^{n}(-1)^{j-1} \frac{\bar{\zeta}_{j}-\bar{z}_{j}}{|\zeta-z|^{2 n}} d \bar{\zeta}[j] \wedge d \zeta
$$

is referred to as the Bochner-Martinelli kernel, cf. [5]. Obviously, $M f$ is a harmonic function in $\mathbb{C}^{n} \backslash S$, and it vanishes at infinity unless $n=1$. Moreover, $M f$ is of finite order growth near $S$, hence $M f$ possesses weak limit values on $S$ both from within and without $S$.

If $z \in S$ then the integral (2.1) no longer exists, for the kernel $U(\zeta, z)$ has a point singularity at $z$ whose order just amounts to the dimension of $S$. Moreover, if $f$ is merely continuous then even the Cauchy principal value of $M f$ may fail to exist, i.e.,

$$
\text { p.v. } M f(z)=\lim _{\varepsilon \rightarrow 0} \int_{\substack{|\zeta \in S\\| \zeta-z \mid \geq \varepsilon}} f(\zeta) U(\zeta, z)
$$

for $z \in S$. where

However, if the function $f$ satisfies Dini's condition at $z$, i.e., $\int_{0}^{1} \frac{\omega_{\theta}(f, z)}{\theta} d \theta<\infty$,

$$
\omega_{\theta}(f, z):=\sup _{\substack{\zeta \in S \\|\zeta-z|<\theta}}|f(\zeta)-f(z)|
$$

is the continuity modulus of $f$ at $z$, then the Cauchy principal value integral (2.2) exists at this point.

Lemma 2.2. If $f \in L_{\mathrm{comp}}^{1}(S)$ satisfies Dini's condition at a point $z \in S$ then the singular integral (2.2) exists, and

$$
\lim _{\varepsilon \rightarrow 0} M f(z \pm \varepsilon \nu(z))=\mp \frac{1}{2} f(z)+\text { p.v. } M f(z) .
$$

Proof. For this and other generalizations of the classical Sokhotskii-Plemelj jump formulas we refer the reader to [5].

Lemma 2.2 shows in particular that the cancellation condition, which is necessary and sufficient for the existence of a singular integral operator, is fulfilled for singular Bochner-Martinelli integral (2.2). From now on we restrict our discussion to this singular integral operator and will write it simply $M_{S} f$. If $S$ is of class $C^{\infty}$ then $M_{S}: C_{\text {comp }}^{\infty}(S) \rightarrow C^{\infty}(S)$ is a polyhomogeneous pseudodifferential operator of zero order on $S$. 


\section{Evaluation of the Symbol}

In this section we evaluate the principal symbol of the singular Bochner-Martinelli integral (2.2). To this end, we identify the cotangent space $T_{z}^{*} S$ of $S$ at a point $z \in S$ with all linear forms on $T_{z}^{*} \mathbb{R}^{2 n}$ which vanish on the one-dimensional subspace of $T_{z}^{*} \mathbb{R}^{2 n}$ spanned by $\nu(z)$. Since $T_{z}^{*} \mathbb{R}^{2 n} \cong \mathbb{R}^{2 n}$, one can actually specify $T_{z}^{*} S$ as the hyperplane through the origin in $\mathbb{R}^{2 n}$ which is orthogonal to the vector $\nu(z)$.

Lemma 3.1. For all $z \in S$ and $\xi \in T_{z}^{*} S$, the symbol of order 0 of the operator $M_{S}$ is equal to

$$
\sigma^{0}\left(M_{S}\right)(z, \xi)=\frac{1}{2} \sum_{j=1}^{n}\left(\nu_{j}(z) \frac{\xi_{n+j}}{|\xi|}-\nu_{n+j}(z) \frac{\xi_{j}}{|\xi|}\right)
$$

Proof. Using Lemma 2.1 we get

$$
\begin{aligned}
M_{S} f(z) & =\frac{1}{\sigma_{2 n}} \int_{S} f(\zeta) \sum_{j=1}^{n} \frac{\bar{\zeta}_{j}-\bar{z}_{j}}{|\zeta-z|^{2 n}} \nu_{c, j}(\zeta) d s(\zeta) \\
& =\int_{S} f(\zeta)\left(2 \sum_{j=1}^{n} \nu_{c, j}(\zeta) \frac{\partial}{\partial \zeta_{j}}\right) G_{2 n}(z-\zeta) d s(\zeta)
\end{aligned}
$$

where $\sigma_{2 n}$ is the area of the $(2 n-1)$-dimensional sphere in $\mathbb{R}^{2 n}$, and

$$
G_{2 n}(z)=\frac{1}{\sigma_{2 n}(2-2 n)} \frac{1}{|z|^{2 n-2}}
$$

is the standard fundamental solution of convolution type of the Laplace operator in $\mathbb{R}^{2 n}$.

Let $\mathcal{G}_{2 n}$ stand for the operator with Schwartz kernel $G_{2 n}(z-\zeta)$ in $\mathbb{R}^{2 n}$. This is a polyhomogeneous pseudodifferential operator of order -2 well known as the Newton potential in $\mathbb{R}^{2 n}$. Its principal symbol is $-|\xi|^{-2}$. The equality (3.1) means that

$$
M_{S} f(z)=\mathcal{G}_{2 n}\left(2 \sum_{j=1}^{n} \nu_{c, j}(\zeta) \frac{\partial}{\partial \zeta_{j}}\right)^{\prime}\left(f \sigma_{S}\right),
$$

where the prime stands for the transposed operator, and $\sigma_{S}$ is the surface layer on $S$.

We thus see that the pseudodifferential $M_{S}$ on $S$ is the restriction to $S$ of the pseudodifferential operator

$$
\Psi=\mathcal{G}_{2 n}\left(2 \sum_{j=1}^{n} \nu_{c, j}(\zeta) \frac{\partial}{\partial \zeta_{j}}\right)^{\prime}
$$

on all of $\mathbb{R}^{2 n}$. This latter is of order -1 and its principal symbol is easily evaluated, namely

$$
\begin{aligned}
\sigma^{-1}(\Psi)(z, \xi) & =\frac{\imath}{|\xi|^{2}} \sum_{j=1}^{n} \nu_{c, j}(z)\left(\xi_{j}-\imath \xi_{n+j}\right) \\
& =\imath \sum_{j=1}^{n} \nu_{c, j}(z) \frac{\xi_{j}-\imath \xi_{n+j}}{|\xi|^{2}}
\end{aligned}
$$


for $z$ in a neighbourhood of $S$ and $\xi \in \mathbb{R}^{2 n}$. A familiar argument now shows that the principal symbol of $M_{S}$ is given by the formula

$$
\begin{aligned}
\sigma^{0}\left(M_{S}\right)(z, \xi) & =\frac{1}{2 \pi} \text { p.v. } \int_{-\infty}^{\infty} \sigma^{-1}(\Psi)(z, t \nu(z)+\xi) d t \\
& =\frac{\imath}{2 \pi} \sum_{j=1}^{n} \nu_{c, j}(z) \text { p.v. } \int_{-\infty}^{\infty} \frac{\left(t \nu_{j}(z)+\xi_{j}\right)-\imath\left(t \nu_{n+j}(z)+\xi_{n+j}\right)}{|t \nu(z)+\xi|^{2}} d t \\
& =\frac{\imath}{2 \pi} \sum_{j=1}^{n} \nu_{c, j}(z) \text { p.v. } \int_{-\infty}^{\infty} \frac{t \overline{\nu_{c, j}(z)}+\left(\xi_{j}-\imath \xi_{n+j}\right)}{t^{2}+2 t\langle\nu(z), \xi\rangle+|\xi|^{2}} d t
\end{aligned}
$$

for all $z \in S$ and $\xi \in \mathbb{R}^{2 n}$ orthogonal to the vector $\nu(z)$. Note that the integral on the right-hand side diverges, however, its Cauchy principal value exists, which is due to the condition $\langle\nu(z), \xi\rangle=0$. We finally obtain

$$
\begin{aligned}
\sigma^{0}\left(M_{S}\right)(z, \xi) & =\frac{\imath}{2 \pi} \sum_{j=1}^{n} \nu_{c, j}(z)\left(\xi_{j}-\imath \xi_{n+j}\right) \int_{-\infty}^{\infty} \frac{1}{t^{2}+|\xi|^{2}} d t \\
& =\frac{\imath}{2|\xi|} \sum_{j=1}^{n} \nu_{c, j}(z)\left(\xi_{j}-\imath \xi_{n+j}\right),
\end{aligned}
$$

which just amounts to

$$
\sigma^{0}\left(M_{S}\right)(z, \xi)=\frac{1}{2} \sum_{j=1}^{n}\left(\nu_{j}(z) \frac{\xi_{n+j}}{|\xi|}-\nu_{n+j}(z) \frac{\xi_{j}}{|\xi|}\right)+\frac{\imath}{2|\xi|}\langle\nu(z), \xi\rangle
$$

showing the lemma.

Note that for $S=\left\{z \in \mathbb{C}^{n}: \Im z_{n}=0\right\}$ Lemma 3.1 gives

$$
\sigma^{0}\left(M_{S}\right)(z, \xi)=-\frac{1}{2} \frac{\xi_{n}}{|\xi|}
$$

which obviously agrees with the symbol of the Hilbert transform on the real axis, i.e., for $n=1$.

Worth mentioning a very simple and transparent geometric interpretation of the symbol $\sigma^{0}\left(M_{S}\right)$. The tangent hyperplane $T_{z} S$ of $S$ at a point $z$ contains the complex plane $T_{\mathbb{C}, z} S$ of real dimension $2 n-2$ determined by the equation $\left(\nu_{c}(z), \zeta-z\right)=0$, where by $(\cdot, \cdot)$ is meant the scalar product in $\mathbb{C}^{n}$. This plane is called the complex tangent plane of $S$ at $z$. The vector $\nu_{c}(z) \in \mathbb{C}^{n}$ spans over $\mathbb{C}$ a complex plane $N_{\mathbb{C}, z} S$ of real dimension 2 in $\mathbb{C}^{n}$, which is called the complex normal plane of $S$ at $z$. The whole space $\mathbb{C}^{n}$ splits into the orthogonal sum $T_{\mathbb{C}, z} S \oplus N_{\mathbb{C}, z} S$, if we put the origin at $z$. The question is now how well is $S$ fit in this complex decomposition. The complex vector $\nu_{c}(z)$ is identified under the complex structure in $\mathbb{R}^{2 n}$ with the real vector $\nu(z)$. Hence, the multiplication of $\nu_{c}(z)$ with $\imath$ puts it in the tangent hyperplane $T_{z} S$. Being the intersection of $T_{z} S$ and $N_{\mathbb{C}, z} S$, the real vector $\imath \nu_{c}(z)=\left(-\nu_{n+1}, \ldots,-\nu_{2 n}, \nu_{1}, \ldots, \nu_{n}\right)$ completes $\nu_{c}(z)$ to an orthonormal basis of $N_{\mathbb{C}, z} S$. The real line in $T_{z} S$ determined by $\imath \nu_{c}(z)$ is well known in complex analysis and is usually referred to as distinguished direction. Lemma 3.1 then shows that

$$
\sigma^{0}\left(M_{S}\right)(z, \xi)=\frac{1}{2|\xi|}\left\langle\imath \nu_{c}(z), \xi\right\rangle
$$


i.e., the value of the principal symbol of $M_{S}$ at $(z, \xi)$ just amounts to half the orthogonal projection of the cotangent vector $\xi \in T_{z}^{*} S$ onto the distinguished direction.

\section{4. $C^{*}$-ALGEBRA}

Suppose $\mathcal{D}$ is a bounded domain in $\mathbb{C}^{n}$ whose boundary is a smooth compact closed hypersurface $S$.

We first note that the operator $M_{S}$ is essentially selfadjoint, i.e., the difference $M_{S}-M_{S}^{*}$ is compact. This is a direct consequence of the fact that the principal symbol of $M_{S}$ is real-valued. Furthermore, it is known that the operator $M_{S}$ is selfadjoint if and only if $S$ is a sphere in $\mathbb{C}^{n}$, cf. [5].

Denote by $\mathcal{A}=\mathcal{A}\left(C(S), M_{S}\right)$ the $C^{*}$-algebra generated by the Bochner-Martinelli integral $M_{S}$, its adjoint operator $M_{S}^{*}$, and by all multiplication operators $a I$ with $a \in C(S)$.

For $n=1$, the algebra $\mathcal{A}\left(C(S), M_{S}\right)$ coincides with the whole algebra of onedimensional singular integral operators, while for $n \geq 2$ it is a proper subalgebra of zero order pseudodifferential operators on $S$.

Theorem 4.1. The $C^{*}$-algebra $\mathcal{A}$ is irreducible.

Proof. In order to establish the theorem we show that the algebra $\mathcal{A}$ does not have any non-trivial invariant subspace. Each invariant subspace of its subalgebra $\{a I: a \in C(S)\} \cong C(S)$ is obviously of the form $V_{\Sigma}=\left\{\chi_{\Sigma} f: f \in L^{2}(S)\right\}$, where $\chi_{\Sigma}$ is the characteristic function of a measurable subset $\Sigma \subset S$ of positive measure. Now, the space $V_{\Sigma}$ is invariant for the entire algebra $\mathcal{A}$ if and only if $\chi_{\Sigma} M_{S}=M_{S} \chi_{\Sigma} I$. In this case the function

$$
\begin{aligned}
\chi_{\Sigma} & =\chi_{\Sigma} \cdot 1 \\
& =\left(\chi_{\Sigma}\left(\frac{1}{2} I+M_{S}\right)\right) 1 \\
& =\left(\left(\frac{1}{2} I+M_{S}\right) \chi_{\Sigma} I\right) 1 \\
& =\left(\frac{1}{2} I+M_{S}\right) \chi_{\Sigma}
\end{aligned}
$$

admits an analytic extension to the domain $\mathcal{D}$ which thus possesses the boundary values

$$
\chi_{\Sigma}(z)= \begin{cases}1, & \text { if } \quad z \in \Sigma, \\ 0, & \text { if } \quad z \in S \backslash \Sigma,\end{cases}
$$

on $S$. Thus, modulo a zero measure set, either $\Sigma=\emptyset$ or $\Sigma=S$. Hence it follows that each invariant subspace of the algebra $\mathcal{A}$ is trivial, being either $\{0\}$ or $L^{2}(S)$, as desired.

Being irreducible, the algebra $\mathcal{A}$ contains non-zero compact operators (for example, commutators $\left.\left[a I, M_{S}\right]\right)$. By Theorem 2.4.9 of [6], it contains the entire ideal $\mathcal{K}$ of compact operators on $L^{2}(S)$.

Denote by $\widehat{\mathcal{A}}=\mathcal{A} / \mathcal{K}$ the Calkin algebra of the algebra $\mathcal{A}$. The essential spectrum of the operator $M_{S}$, i.e., the spectrum of the image $\widehat{M}_{S}$ of $M_{S}$ in the Calkin algebra, coincides obviously with the range of its principal symbol. By (3.3), the essential spectrum of $M_{S}$ just amounts to the interval $[-1 / 2,1 / 2]$, and thus, by the standard 
functional calculus, the $C^{*}$-subalgebra of $\widehat{\mathcal{A}}$ generated by $\widehat{M}_{S}$ is isomorphic and isometric to $C[-1 / 2,1 / 2]$.

The description of the Calkin algebra $\widehat{\mathcal{A}}$ can be obtained either by using the familiar localization technique or by deducing the result from the well-known description of the Calkin algebra for the $C^{*}$-algebra of zero order pseudodifferential operators on the surface $S$.

Theorem 4.2. The Calkin algebra $\widehat{\mathcal{A}}$ of the algebra $\mathcal{A}=\mathcal{A}\left(C(S), M_{S}\right)$ is isomorphic and isometric to $C(S \times[-1 / 2,1 / 2])$. Under this isomorphism, the homomorphism $\pi: \mathcal{A} \rightarrow \widehat{\mathcal{A}}$ is generated by the following mapping of generators of the algebra $\mathcal{A}$ :

$$
\begin{array}{lrll}
\pi: & a I & \mapsto a(z), & z \in S ; \\
\pi: & M_{S} & \mapsto \sigma, & \sigma \in[-1 / 2,1 / 2] .
\end{array}
$$

Note that a zero order pseudodifferential operator $\Psi$ belongs to the algebra $\mathcal{A}$ if and only if its principal symbol has the form

$$
\sigma^{0}(\Psi)(z, \xi)=a\left(z, \frac{1}{2|\xi|}\left\langle\imath \nu_{c}(z), \xi\right\rangle\right)
$$

for a suitable function $a \in C(S \times[-1 / 2,1 / 2])$.

\section{The Szegö PROJection}

Let $\mathcal{D}$ be a strictly pseudoconvex domain in $\mathbb{C}^{n}$, and let $S$ be its boundary. As in Section 2, we write $d s$ for the surface measure on $S$, and consider $L^{2}(S)$ with respect to this measure.

Moreover, we denote by $H^{2}(S)$ the Hardy space on $S$, that is, the subspace of $L^{2}(S)$ consisting of all functions admitting an analytic continuation to the domain $\mathcal{D}$. Let $P_{S}$ stand for the orthogonal projection of $L^{2}(S)$ onto $H^{2}(S)$, called also Szegö projection.

By Lemma 2.2, the limit values on $S$ of the Bochner-Martinelli integral from within $S$ are related to the singular integral by the formula $M_{-}=(1 / 2) I+M_{S}$. In [8] the iterations of $M_{-}$are proved to converge in the strong topology of $\mathcal{L}\left(L^{2}(S)\right)$ to the Szegö projection $P_{S}$ in case $S$ is the boundary of a ball in $\mathbb{C}^{n}$. The question arises whether this result can be extended to arbitrary strictly pseudoconvex domains $\mathcal{D}$.

Using (3.3), we may readily evaluate the principal symbol of iterations $M_{-}^{N}$ for all $N=1,2, \ldots$. This yields

$$
\begin{aligned}
\sigma^{0}\left(M_{-}^{N}\right)(z, \xi) & =\left(\frac{1}{2}+\frac{1}{2|\xi|}\left\langle\imath \nu_{c}(z), \xi\right\rangle\right)^{N} \\
& =\frac{1}{2^{N}}\left(1+\left\langle\imath \nu_{c}(z), \frac{\xi}{|\xi|}\right\rangle\right)^{N}
\end{aligned}
$$

whenever $z \in S$ and $\xi \in \mathbb{R}^{2 n}$ is orthogonal to the normal vector $\nu(z)$. From (5.1) we deduce by the Schwarz inequality that

$$
\lim _{N \rightarrow \infty} \sigma^{0}\left(M_{-}^{N}\right)(z, \xi)= \begin{cases}1, & \text { if } \xi=t \imath \nu_{c}(z), t>0, \\ 0, & \text { otherwise, }\end{cases}
$$

for all $z \in S$. Moreover, this convergence is uniform on each subset of $T^{*} S$ of the form $z \in S$ and $|\xi /| \xi|-\imath \nu(z)| \geq \varepsilon$, with $\varepsilon>0$. 
Note that $\left\{(z, \xi): \xi=t \imath \nu_{c}(z), t>0\right\}$ is just the symplectic submanifold of $T^{*} S$, on which the symbols of generalized Toeplitz operators live, cf. $[2, \S 1]$. In [2], the covector $\imath \nu_{c}(z)$ is written as one-form

$$
-\iota^{*} \Im \frac{\bar{\partial} \varrho(z)}{|\bar{\partial} \varrho(z)|}
$$

where $\iota: S \hookrightarrow \mathbb{C}^{n}$ is the inclusion map.

The equality (5.2) shows that the limit of $\sigma^{0}\left(M_{-}^{N}\right)$ is exactly the principal symbol of the Szegö projection $P_{S}$. Since the generalized Toeplitz operators of negative order induce compact operators on $L^{2}(S)$, cf. ibid., (5.2) gives us a microlocal version of Romanov's theorem [8] which is also valid for arbitrary strictly pseudoconvex surfaces.

Theorem 5.1. Assume that $S$ is the boundary of a strictly pseudoconvex domain. Then

$$
\lim _{N \rightarrow \infty}\left((1 / 2) I+M_{S}\right)^{N}=P_{S}+K_{S}
$$

in the strong topology of $\mathcal{L}\left(L^{2}(S)\right)$, where $K_{S}$ is a compact operator.

As but one direct and simple consequence of Theorem 5.1 we mention that

$$
\begin{aligned}
P_{S}\left(2 M_{S}\right)+K_{S}^{\prime} & =\left(2 M_{S}\right) P_{S}+K_{S}^{\prime \prime} \\
& =P_{S}\left(2 M_{S}\right) P_{S}+K_{S}^{\prime \prime \prime} \\
& =P_{S},
\end{aligned}
$$

where $K_{S}^{\prime}$ and $K_{S}^{\prime \prime}$ are compact operators, and $K_{S}^{\prime \prime \prime}=K_{S}^{\prime} P_{S}=P_{S} K_{S}^{\prime \prime}$. While for $S$ being the boundary of a ball one has exactly

$$
\begin{aligned}
P_{S}\left(2 M_{S}\right) & =\left(2 M_{S}\right) P_{S} \\
& =P_{S}\left(2 M_{S}\right) P_{S} \\
& =P_{S} .
\end{aligned}
$$

In conclusion we mention as well that if $\Psi$ is a pseudodifferential operator in $\mathcal{A}$ with principal symbol (4.1) then $P_{S} \Psi P_{S}=P_{S}(a(z, 1 / 2) I) P_{S}$ holds modulo compact operators. 


\section{REFERENCES}

1. Boutet De Monvel, L., On the index of Toeplitz operators of several complex variables, Inventiones Math. 50 (1979), 249-272.

2. Boutet de Monvel, L. and Guillemin, V., The Spectral Theory of Toeplitz Operators, Princeton University Press, Princeton, New Jersey, 1981.

3. Guillemin, V., Toeplitz operators in $n$ dimensions, Integral Equations Operator Theory 7 (1984), no. 2, 145-205.

4. Günter, N. M., Potential Theory and Its Application to Fundamental Problems of Mathematical Physics, Gostekhizdat, Moscow, 1953, 415 p.

5. Kytmanov, A. M., The Bochner-Martinelli Integral, and Its Applications, Birkhäuser Verlag, Basel et al., 1995.

6. Murphy, G. I., $C^{*}$-Algebras and Operator Theory, Academic Press, Inc., Boston et al., 1990.

7. Nagel, A. and Stein, E. M., Lectures on Pseudo-Differential Operators, Princeton University Press, Princeton, New Jersey, 1979.

8. Romanov, A. V., Spectral analysis of Martinelli-Bochner integral for ball in $\mathbb{C}^{n}$, and its applications, Functional Anal. Appl. 12 (1978), no. 3.

Institute of Mathematics, University of Potsdam, PO Box 6015 53, 14415 Potsdam, GERMANY

E-mail address: tarkhanov@math.uni-potsdam.de

Departamento de Matemáticas, CinvestaV del I.P.N., Apartado Postal 14-740, 07000 MÉxico, D.F., MÉxico

E-mail address: nvasilev@duke.math.cinvestav.mx 